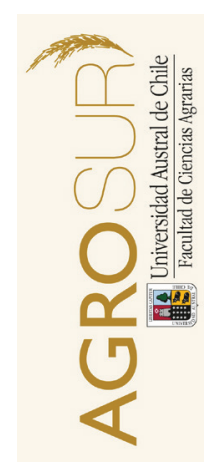

\title{
Descripción del manejo productivo del jabalí (Sus scrofa L.) en un sistema semi-extensivo en la zona sur de Chile
}

\author{
Description of the productive management of the European wild boar \\ (Sus scrofa L.) under a semi-extensive system in the South of Chile
}

\section{Keywords: \\ Exotic Species \\ Farm \\ Production \\ Breeding}

A R T I C L E INFO

Original Research Article,

Animal Science

*Corresponding author:

Suzanne Hodgkinson

E-mail address:

shodgkin@uach.cl

\section{Álvarez, A. ${ }^{a}$, Hodgkinson, $\mathrm{S}^{b^{*}}$, Flores, $\mathrm{P}^{b}$}

${ }^{a}$ Escuela de Agronomía Facultad de Ciencias Agrarias, Universidad Austral de Chile.

${ }^{b}$ Instituto de Producción Animal, Facultad de Ciencias Agrarias, Universidad Austral de Chile. Casilla 567 Valdivia, Chile.

\section{RESUMEN}

El objetivo del presente estudio fue describir la producción de jabalí europeo en un predio comercial usando un sistema semi-extensivo, que es típico del sur de Chile. El estudio de caso se llevó a cabo en un plantel comercial en San José de la Mariquina, Chile. La metodología utilizada para recoger los datos incluyó observaciones realizadas durante las visitas al Fundo en las diferentes etapas de producción. El administrador y los propietarios fueron entrevistados y la información recogida fue corroborada a través de las observaciones realizadas durante las visitas. Este predio ha producido jabalí europeo por más de 12 años en un área de 3,5 hectáreas. El rebaño incluye actualmente un verraco de cría y 14 hembras reproductoras.

Palabras clave: Especie Exótica, Predio, Producción, Crianza.

\section{INTRODUCCIÓN}

El jabalí forma parte de una de las tantas especies exóticas que se encuentran en Chile y como tal, podría representar un peligro para las especies endémicas debido a la transmisión de enfermedades, o por el daño a cultivos y la degradación del suelo (Novoa, 2002).

La ley de caza $\mathrm{N}^{\circ} 19.473$ del Ministerio de Agricultura obliga a la inscripción de estos animales. Sin embargo, la mayoría de los criaderos no han sido registrados. El censo agrícola ganadero que constituye la fuente primaria de datos que permite caracterizar la estructura y funcionamiento del sector agropecuario y forestal señala que para el año 2007 se contabilizó 4.005 cabezas de jabalís con una base de informantes de 245 predios (INE, 2012). Estos animales se encuentran desde la II Región hasta la XI Región, incluyendo la
XIV Región (anteriormente formando parte de la X Región). Skewes y Morales (2006) indicaron que más del $70 \%$ de los criaderos cuentan con menos de 50 animales. El $60 \%$ de los animales tienen procedencia de otros criaderos, el $30 \%$ es capturado del medio silvestre y el $10 \%$ se obtiene de ambos orígenes. En la VI Región se encuentra el mayor número de animales mientras que en IX y XIV se encuentra el mayor número de criaderos. En relación a la pureza genética del jabalí aproximadamente el $12 \%$ de los criaderos posee certificación de que sus animales son puros.

Los jabalies se crían dentro de dos sistemas productivos. El primero es un sistema de producción extensiva, el cual simula las condiciones naturales donde vive. El segundo es semi-extensivo, el cual se desarrolla al aire libre (Skewes y Morales, 2006) que tiene menor superficie por animal, con provisión de refugio y corral 
e incluye dentro de la alimentación el aporte de granos (Nixdorf y Barber, 2001). En condiciones salvajes su dieta se basa sobre un $90 \%$ en material vegetal y hasta un $10 \%$ en alguna fuente de origen animal (FournierChambrillon et al., 1995).

Se ha señalado que manejos básicos como programas de medicina preventiva no se consideran necesarios porque se tratan de animales silvestres (Skewes y Morales, 2006). Los manejos realizados se limitan a tratamientos antiparasitarios o algunas vacunas, pero no se posee un programa establecido para ello. En el sistema semi-extensivo se trata de compatibilizar las características propias del animal y las ventajas de este sistema, como por ejemplo una baja inversión inicial, utilización de suelos con praderas de menor calidad nutricional, bajo impacto ambiental y escasa mano de obra. Entre la X y XIV regiones es donde se destina mayor superficie de pradera por animal debido a que ésta es el principal recurso disponible.

En cuanto a la infraestructura, es necesario crear refugios para que se protejan de las condiciones climáticas en invierno, y para el verano crear un charco de barro para que se refresquen. Según las normas del SAG, los cercos deben tener un mínimo de 1,2 m de altura y extenderse $0,45 \mathrm{~m}$ bajo el nivel del suelo debido a que los jabalíes tienden a cavar en el suelo y pueden escapar del potrero. Además, los cercos protegen a los animales más jóvenes y pequeños de posibles depredadores. El cerco debe poseer una malla con tejido cerrado y la distancia entre postes debe ser máxima de $5 \mathrm{~m}$. Las instalaciones deben tener además, infraestructura que permita separar a los animales para poder realizar manejos básicos como destete, pesaje y cargadero para la posterior venta de los animales (Nixdorf y Barber, 2001).

Actualmente hay muy poca información sobre los manejos utilizados en planteles comerciales de jabalíes. Esta escasez de información dificulta el trabajo de productores, que no cuenten con datos para comparar sus índices productivos. Por lo tanto, el objetivo de este trabajo fue describir y analizar un estudio de caso, en base a sustentos teóricos, la productividad y el manejo realizados en la crianza de jabalíes de un fundo específico de la XIV región uno de los primeros criaderos funcionando en Chile, siendo establecido en 1998.

\section{MATERIAL Y MÉTODOS}

\section{Localización y duración del estudio}

El estudio se realizó en el Fundo Kutrehue, ubicado en la comuna de San José de la Mariquina, en la XIV Región de Los Ríos. Las observaciones y entrevistas se llevaron a cabo entre mayo de 2012 y abril de 2013. El predio posee acceso cercano a la carretera principal y cuenta con 135 ha donde principalmente se desarro- llan rubros de producción de carne bovina y cultivos forestales. La actividad de crianza de jabalíes se desarrolla en 3,5 ha.

\section{Información recolectada}

Debido a que el Fundo no tiene un sistema desarrollado para mantener registros de producción, el estudio fue realizado en base a observaciones a través de cuatro visitas al predio en diferentes etapas de la producción. En conjunto se efectuaron preguntas previamente elaboradas en cuanto a la producción y prácticas de manejo del lugar al encargado de los animales y a los dueños del predio. Los datos fueron corroborados a través de las observaciones realizadas durante las visitas.

A partir de la información recolectada se realizó una descripción del predio la cual consistió en: a) Objetivo productivo del predio (fines lucrativos, entretención, productivos o de otro interés); b) Sistema de producción (tipo de sistema utilizado, recursos disponibles, características de la pradera incluyendo especies presentes, alimentación disponible, insumos e infraestructura); c) Características de los animales (número de reproductores machos y hembras, edad y peso, animales mestizos o puros, origen de los reproductores nuevos, criterios de reposición y de selección de reproductores, alimentación, cantidad de animales en crecimiento y destino de éstos); d) Prácticas de manejo y factores de productividad.

Además se realizó una descripción de las etapas productivas como: encaste, gestación, parición, lactancia, destete, crecimiento y engorda.

En la descripción de los datos recolectados se contemplaron principalmente las características propias del Fundo Kutrehue (animales, sistema productivo, infraestructura y espacio físico designado para los animales). Además cuando existieron los datos necesarios, se calcularon parámetros de desempeño promedio del criadero, tales como: relación hembra/macho (número de machos utilizados por hembra a encastar); índice de fertilidad de hembras (porcentaje de hembras que producen una camada por año); mortalidad durante la gestación (\%) [(número de hembras muertas en gestación/ número de total hembras que encastaron en gestación) x 100]; número de jabatos nacidos vivos; mortalidad de jabatos (\%) [(número de jabatos muertos/número total de jabatos nacidos" x 100]; número de jabatos/ hembra/año: (determinado por número de camadas/ año y número de jabatos/camada); ciclo productivo de la hembra (días de gestación + días de lactancia + días post-parto); peso de la hembra al encaste (kg); peso de la hembra al final de la gestación (kg); peso de jabatos al nacer ( $\mathrm{kg})$; peso de destete (kg); tasa de crecimiento ([peso final-peso inicial]/días); índice de conversión alimenticia ( $\mathrm{kg}$ de materia seca consumido/kg de peso ganado); edad (días) y peso de término (kg). 
Los datos recopilados a través de la observación junto con los resultados y respuestas de las preguntas previamente formuladas se analizaron con la información ya existente sobre jabalíes. En el caso de no encontrar información para algún dato evaluado se comparó con información de cerdo doméstico (Sus scrofa domestica) producidos en un sistema semi-extensivo.

\section{RESULTADOS Y DISCUSIÓN}

\section{Antecedentes productivos del fundo}

El Fundo Kutrehue posee 135 ha en las cuales se desarrollan diferentes actividades agropecuarias y forestales. Desde el año 1998 destinaron 3,5 ha a un criadero para la producción de jabalíes y dos años después se inscribieron como criadero ante el Servicio Agrícola Ganadero (SAG) con el objetivo de generar animales para la comercialización de carne de animales de 6 a 7 meses de edad (dispuesto a variabilidad). Hoy en día los productores buscan aumentar la ganancia de peso a través de manejos agronómicos y también esperan adquirir un macho genéticamente puro. No se quiere aumentar el número de reproductores ni crías ya que poseen un solo comprador.

El sistema productivo utilizado es el semi-extensivo ya que se busca simular en cierta manera el ambien- te natural donde viven. Los animales se alimentan del recurso disponible, en este caso praderas formadas principalmente por Lolium multiflorum Lam., Trifolium pratense L. y algunas especies de hoja ancha tales como Taraxacum officinale Weber e Hipochaeris radicata $\mathrm{L}$. entre otras, usando un sistema de pastoreo continuo. Junto con ello, se les suplementa con concentrado de cerdo doméstico y subproductos de la agroindustria. Las 3,5 ha se encuentran rodeadas por un cerco de madera con malla de alambre con una altura de 1,2 m. Además es acompañado por un cerco vivo de Pinus radiata D. Don (esta condición es una exigencia del SAG debido a que los jabalíes son considerados una plaga). Este cerco de árboles refuerza el aislamiento de los animales con el resto del entorno.

\section{Uso del suelo e Infraestructura}

Las 3,5 ha se encuentran divididas en secciones (Figura 1). La primera sección se encuentra divida por un cerco eléctrico en las secciones 1 y 1 A. La sección 1 posee un encino (Querqus ilex L.) y 4 castaños (Castanea sativa Mill.) los que aportan una cobertura mínima para el invierno, sombra para el verano y alimento con sus semillas. Además hay un comedero de madera donde depositan el suero, un segundo comedero el cual posee una base de cemento y cerco con madera con una

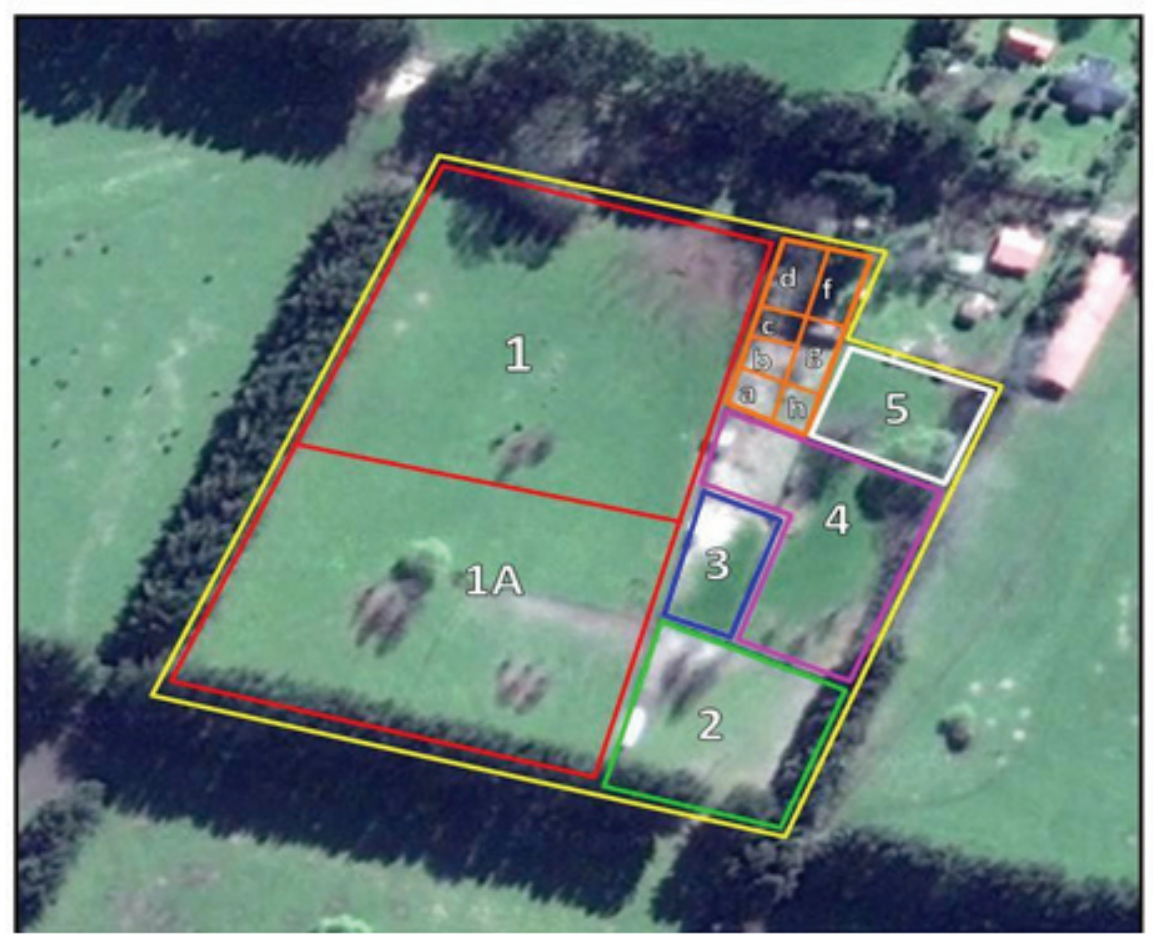

Figura 1. Secciones en las que se dividen las 3,5 ha del criadero de jabalíes, en el Fundo Kutrehue. Secciones 1, 1 A, 2, 3, 4, 5, a, b, c, d, f, g, h.

Figure 1. Sections dividing the 3.5 ha of the European wild boar farm, Kutrehue Farm. Section 1, 1 A, 2, 3, 4, 5, a, b, c, d, f, g, h. 
altura de 1,5 m. Este comedero posee una pequeña entrada la cual restringe el paso a los animales grandes, permitiendo el paso solo a jabatos, aquí se deposita principalmente algún tipo de grano.

La sección 1A contiene 4 encinos, 4 castaños y 3 bebederos tipo chupón. En verano bajo estos bebederos los jabalíes forman un charco para refrescarse del calor. Además posee una estructura abierta de madera con techo de lata la cual funciona como refugio o nidales según la época del año y a los animales que allí se encuentren.

Las secciones 2 y 3 son donde se mantienen los animales rezagados, los cuales no han alcanzado el peso y la conformación física visualmente esperada, por lo que van quedando atrás con respecto al grupo, a medida que van ganando peso se pueden reincorporar a un grupo homogéneo de jabalíes con un peso similar, de lo contrario seguirán rezagados hasta que alcancen un peso óptimo para enviar a sacrificio. La sección 2 posee 4 encinos, 2 bebederos tipo chupón, un charco, un refugio y un comedero de madera. La sección 3 posee 3 bebederos tipo chupón y un refugio. La sección 4 posee 3 árboles de encino, 6 bebederos tipo chupón y 2 refugios, mientras que la sección 5 es un potrero para pradera rezagada conformada por 2 encinos, 3 bebederos tipo chupón y un refugio. Las secciones a, b y c son pequeños potreros sin praderas, con un bebedero y un refugio cada uno. Las secciones $\mathrm{d}$, $\mathrm{f}$, g y $\mathrm{h}$ son pequeños potreros con pradera rezagada para rotar animales de la sección 3, solo poseen bebederos.

En el fundo además existe una zona de espera donde pesan los animales en una balanza (con capacidad de $1000 \mathrm{~kg}$ ) en grupos de diez, y posteriormente los suben por el cargadero al camión con destino a faena en un matadero autorizado.

\section{Descripción de los animales}

El sistema cuenta con 14 hembras reproductoras de hasta 7 años de edad y de $80 \mathrm{~kg}$ de peso vivo. Hay un macho reproductor de 7 años de edad con $180 \mathrm{~kg}$ de peso el cual se pretende vender para su posterior cambio por uno con genotipo puro. Años atrás la cantidad de reproductores era mayor, pero hoy el sistema está simplificado y se trabaja con una cantidad mínima de animales. Tanto las hembras como el macho no poseen certificado de pureza y se duda de que los animales sean genéticamente puros. Su comprador no exije que los animales sean certificado como raza pura. Sin embargo, se busca conformación física y que las características fenotípicas de los reproductores sean lo más similar a un jabalí puro al momento de comprar o seleccionar un animal para reemplazo. El origen de los nuevos animales por lo general es del mismo predio, sobre todo en el caso de las hembras. El macho proviene de otro criadero de jabalíes para disminuir o evitar la consanguinidad, ambas selecciones se realizan visualmente buscando características propias del jabalí. La alimentación para ellos se basa en un estimado $80 \%$ de pradera y $20 \%$ de otros alimentos sujetos a disponibilidad (Cuadro 1).

En cuanto a los animales jóvenes, se estima que con esa cantidad de reproductores se manejan entre 60 a 80 animales por año en etapa de crecimiento y engorda, dependiendo del promedio de crías nacidas por cada hembra reproductora. El destino de estos animales es venderlos con un peso aproximado de $50 \mathrm{~kg}$ y se llevan a un matadero ubicado en la cuidad de Chillán el cual es exclusivo para carnes exóticas. Este matadero es el de acceso más cercano. El peso obtenido de la canal varía entre los 35 y $40 \mathrm{~kg}$.

Cuadro 1. Subproductos de la agroindustria e insumos adicionales usados en la alimentación de jabalíes en el Fundo Kutrehue.

Table 1. By products of the agroindustry and additional inputs used to feed European wild boars in Kutrehue Farm.

\begin{tabular}{lll}
\hline Tipo de alimento* & Época del año & Observaciones \\
\hline Papas & Octubre-Enero & Para todos los animales \\
Manzana & Marzo-Abril & Para todos los animales \\
Zanahoria & Marzo-Septiembre & Para todos los animales \\
Avena & Septiembre-Octubre & Jabatos desde 1 semana de edad \\
Suero de queso & Todo el año & Para todos los animales \\
Cabezas de pescado & Esporádico & Para todos los animales \\
Desechos de fabricación de chicha & Marzo-Abril & Para todos los animales \\
Concentrado "Champión" & Noviembre-Abril & Animales en crecimiento y engorda \\
Semillas de encino y castaño & Diciembre-Abril & Para todos los animales, especialmente para \\
\hline
\end{tabular}

*Todos los alimentos están sujetos a variabilidad la cual dependerá del precio del producto y su disponibilidad durante el año; el origen y los productos aportados pueden ir variando año tras año. 


\section{Etapas de la producción}

\section{Fase de cría}

El encaste se realiza en época de otoño entre los meses de marzo y abril (Figura 2). Se reúnen a los reproductores por dos meses en la sección 1 . Actualmente se utilizan 14 hembras y 1 macho. La monta es de tipo natural. No se realizan manejos veterinarios. El recambio de hembras se realiza cada 2 o 3 años y el macho se cambia cada 4 años.

La gestación dura 115 días y según el mes en el que las hembras hayan quedado preñadas los partos se producirán a partir del mes de agosto. No utilizan método para confirmar la preñez y por lo general el $100 \%$ de las hembras quedan preñadas. Hasta el momento reportan que no se ha detectado un caso de enfermedad o complicación durante esta etapa y tampoco aplican suplementos alimenticios como vitaminas o sales minerales. La dieta sigue siendo $80 \%$ en base a praderas y $20 \%$ alimentos de oportunidad. El refugio es utilizado para dormir ya que las protege de las malas condiciones del invierno. La hembra aumenta $15 \mathrm{~kg}$ de peso en este periodo, por lo que alcanzarían pesos de hasta $100 \mathrm{~kg}$. Nunca ha ocurrido la muerte de alguna hembra durante este periodo.

Las pariciones ocurren en la sección 1 desde el mes de Agosto hasta fines de Septiembre, en época de primavera, lo que coincide con el momento en que las praderas obtienen su nivel máximo de producción de materia seca. Algunas hembras utilizan el refugio como nidales al momento de parir. Las hembras paren con una distancia mínima de 5 metros entre ellas y las que no usan un refugio toman ramas, hojas y fardos de paja proporcionados por el productor, las que acomodan formando un nido que les proporciona protección del medio ambiente y aislamiento para los recién nacidos.

Informan que no han tenido problemas con la parición de las hembras, por lo cual el parto es totalmente natural sin ninguna intervención. La alimentación es la misma que es otorgada en la gestación, pero a menudo las hembras no se levantan a comer, por lo que hay que proporcionar alimento cercano a éstas.

El único manejo pre-parto es el aporte de materiales para la formación de sus nidos. Los restos orgánicos producto del parto no son retirados, quedan en el lugar y luego se descomponen. Se obtienen en promedio 5,5 jabatos por hembra, con un peso estimado que llega hasta $1 \mathrm{~kg}$ por cría. Ha ocurrido el nacimiento de animales muertos y muertes post-parto, sin embargo estas no han sido contabilizadas, ya que en este periodo la hembra se torna muy agresiva y territorial, por lo cual es mejor no acceder a su territorio. Se desconocen las causas de muerte. Tampoco se puede asegurar el consumo de calostro debido al mismo motivo.

Se obtiene una camada por hembra al año. No tienen planes de aumentar esa cifra, debido a que en el pasado cuando tenía una segunda camada en otoño, el porcentaje de mortalidad al momento del parto era muy elevado por las condiciones climáticas. Cabe destacar que las madres primerizas obtienen camadas más reducidas y con jabatos de menor peso. No se realiza un registro de los nuevos individuos.

La lactancia dura aproximadamente 2 meses. Al menos durante las primeras 2 semanas los jabatos solo

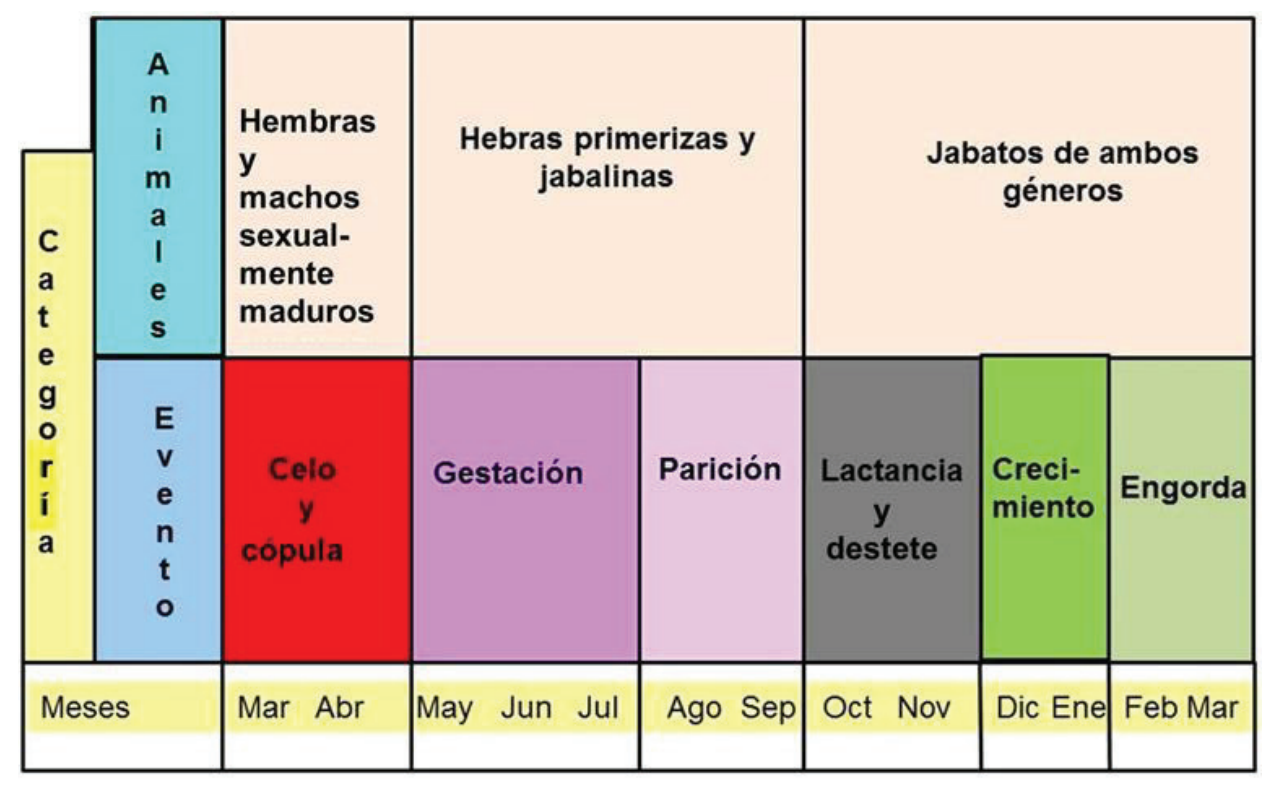

Figura 2. Ciclo productivo anual de la crianza de jabalíes en el Fundo Kutrehue.

Figure 2. Annual productive cycle of European wild boar reared in Kutrehue Farm. 
consumen leche materna debido a que no se separan de la madre y se encuentran la mayor parte del tiempo escondidos en el nido. Luego los jabatos salen del nido a buscar alimento en la pradera y seguir a las madres. Con el comedero de acceso exclusivo a los jabatos, se les alimenta con avena para que comiencen a acostumbrar su sistema digestivo a los alimentos sólidos. El peso aproximado de las crías al final de la etapa de lactancia es entre de 10 a $12 \mathrm{~kg}$. En cuanto a la hembra sus requerimientos nutricionales aumentan debido a la producción de leche y los nutrientes consumidos al parecer son menores a los que necesita. Las hembras llegan a pesos entre 50 a 60 kilos al final de la lactancia.

\section{Fase de crecimiento}

Los jabatos se destetan de las madres de forma gradual y autónoma, una vez que éstos exploran el lugar y prueban los alimentos disponibles. Cuando tienen aproximadamente dos meses de edad, los jabatos son llevados a la sección a, b o c, donde son separados completamente de la madre y se realizan los manejos de desparasitación vía oral (Ivermectina ${ }^{\circledR}$ en polvo mezclada con suero), postura de anillos en la nariz y una dosis de $200 \mathrm{mg}$ de hierro aportado de forma oral. Al cabo de un par de días post destete mediante inspección visual, se escogen los que poseen mejor conformación física y mayor peso para posteriormente llevarlos a la Sección 3.

Los animales seleccionados entran en una etapa llamada "crecimiento". Finalizan la etapa con un peso aproximado de $20 \mathrm{~kg}$. No se hace distinción de género ya que no hay grandes diferencias entre la calidad y sabor de la carne, además la faena se realiza antes de llegar a pubertad por lo que los animales no se aparean durante esta etapa. Durante este periodo se comienza la alimentación con concentrado comercial (Champion $囚$ ) para engorda de cerdo doméstico. Cada animal consume aproximadamente $1 \mathrm{~kg}$ por día. Junto con ello siguen pastoreando y comiendo alimentos de oportunidad.

\section{Fase de engorda}

Luego de 3 meses aproximadamente (alrededor de los 5 meses de edad) se realiza una segunda estimación visual de peso y conformación donde se crea un segundo grupo el cual se lleva a engorda en las secciones 4 o 5. Los animales con un peso vivo y conformación adecuada se juntan con los animales en la sección 2 (animales rezagados).

La engorda finaliza entre los meses de marzo a abril, con 6 a 7 meses de edad y la alimentación es la misma que en la etapa de crecimiento. Los animales tienen un peso de término que varía entre los 45 a $50 \mathrm{~kg}$ el cual se considera óptimo para llevarlos a sacrificio. Solo po- seen un comprador el cual les paga $\$ 5.000$ por kg de carne. El peso obtenido de canal es hasta $40 \mathrm{~kg}$.

\section{Bienestar animal}

Con respecto a bienestar animal, no existen signos evidentes de comportamientos agresivos o estereotípicos. Se desconoce el grado de estrés sufrido por los animales durante el periodo de traslado a la faena.

\section{Índices y parámetros}

No se pudo calcular la mortalidad de jabatos recién nacidos, la mortalidad post parto, ni el peso de los jabatos al destete, debido a territorialidad de la hembra durante ese periodo, pero no permite el acercamiento de trabajadores a la cría. El índice de conversión de alimento tampoco es posible calcularlo ya que se haría bajo premisas no reales al no saber las cantidades exactas de alimentos consumidos y peso del animal. Los índices calculados son presentados en Cuadro 2.

Cabe destacar que en general los manejos realizados y las épocas del año en la cual se desarrollan las etapas de crianza y producción de jabalíes son muy similares a las condiciones señaladas por Novoa (2002) y JABALÍCHILE (2006). Al juzgar visualmente se puede apreciar que los animales poseen más espacio del necesario y su comportamiento social parece sano. Junto con ello, el criadero lleva años en el negocio, por lo que se denota experiencia y aprendizaje de los productores con respecto al cuidado y bienestar de sus animales. Además aprovechan los desechos de productores agrícolas vecinos como alimento para jabalíes, y se utiliza un sitio con praderas de menor calidad en composición nutricional por la presencia de especies consideradas no deseadas para el sistema de producción de carne, por lo que se aprovecha muy bien el espacio y los recursos disponibles para este rubro.

Con respecto a los animales, se desconoce la pureza genética de los jabalíes que componen el criadero del Fundo Kutrehue, por lo que si quisieran mejorar las cualidades propias y características de la carne de jabalí y además, proveer de un mejor producto, se sugiere la adquisición de un macho puro certificado. Actualmente seleccionan las hembras reproductoras del criadero que fenotípicamente tengan las cualidades propias de un jabalí puro o cualidades por las cuales sean consideradas buenas madres.

La relación hembra:macho utilizada según Novoa (2002) es 10:1 en Chile y 15:1 en Canadá (en un sistema más intensivo), en este criadero se utiliza una relación 14:1. Se destaca que este fundo utiliza un periodo de dos meses para la realización del encaste por lo que el $100 \%$ de sus hembras quedan preñadas, lo que es óptimo, por lo que la compra de un segundo macho se volvería necesaria solo en el caso que quisieran redu- 
Cuadro 2. Resumen de los parámetros productivos observados en el criadero de jabalíes, Fundo Kutrehue.

Table 2. Summary of the productive parameters of the European wild boar breeding farm, Farm Kutrehue.

\begin{tabular}{ll}
\hline Relación hembra/macho & $14: 1$ \\
Recambio de hembras & Cada 2 a 3 años \\
Recambio de machos & Cada 4 años \\
Mortalidad durante la gestación & $0 \%$ \\
Número de jabatos nacidos vivos & 5,5 promedio \\
Ciclo productivo de la hembra & 365 días \\
Peso de la hembra al momento de encaste & $80 \mathrm{~kg} *$ \\
Peso de la hembra al final de la gestación & $95-100 \mathrm{~kg} *$ \\
Peso de la hembra final de la lactancia & $50-60 \mathrm{~kg} *$ \\
Promedio camada por hembra/año & 1 \\
Peso de jabatos al nacer & Hasta $1.000 \mathrm{~g}$ \\
Peso destete & $10-12 \mathrm{~kg}$ \\
Duración de lactancia & $2 \mathrm{meses} *$ \\
Peso al salir de la etapa de crecimiento & $20 \mathrm{~kg} *$ \\
Edad a la faena & 6 a 7 meses* \\
Peso de sacrificio & $45-50 \mathrm{~kg} *$ \\
Peso de canal (con cabeza) & $35-40 \mathrm{~kg}$ \\
Precio de venta de carne & $\$ 5.000 / \mathrm{kg}$ \\
Tasa de crecimiento & $166 \mathrm{~g} / \mathrm{día} *$ \\
\hline nacimiento hasta crecimiento) crecimiento (crecimiento a faena) & $277 \mathrm{~g} / \mathrm{día*}$ \\
\hline
\end{tabular}

*Todos estos cálculos fueron realizados a través de datos recolectados con estimaciones visuales realizadas por el encargado el criadero, los datos no son exactos.

cir ese tiempo o si desearan aumentar el número de hembras reproductoras, de lo contrario solo sería un gasto innecesario y un animal más que alimentar. Sin embargo, es necesario renovar el macho a tiempo para prevenir problemas de consanguinidad.

La superficie destinada para la crianza de jabalíes sería suficiente para una mayor cantidad de animales que el número de animales que poseen actualmente. Este se evidencia ya que no consumen toda la pradera producida. Sin embargo, en este caso los productores no desean aumentar la cantidad de animales debido a que solo poseen un comprador y sería muy riesgoso no concretar la venta de los animales. Por lo tanto se plantea que una opción puede ser ampliar los clientes buscando más compradores o asociarse con otros productores para generar mayor volumen de producto y llegar a otros mercados, por ejemplo exportación a países europeos. Otra opción sería introducir animales de otras especies como terneros, caballos u ovejas para aprovechar la materia seca sobrante de la pradera. Cabe destacar que los productores por lo general mantienen corderos en el mismo sector de los jabalíes en primavera cuando sobra pradera para los jabalíes.

Para aumentar la eficiencia y ganancia de peso de los animales se sugiere que exista un sistema de registro con respecto a los alimentos de oportunidad que se dan para complementar la dieta. Para confirmar si se aumentan las ganancias de peso o si las conversiones de alimento mejoran, se consideraría óptimo pesar a los animales al menos dos veces antes de ir a sacrificio. Para ello también es necesario, y se recomienda un sistema de registro para los pesajes y con las fechas de este evento. El primer pesaje se sugiere al momento del destete para poder seleccionar los animales que entren en etapa de crecimiento y así poder aportar la dieta necesaria para esa etapa. El segundo pesaje se podría realizar al final de la etapa de crecimiento (aproximadamente $20 \mathrm{~kg}$ ) para seleccionar los animales que pasarán a etapa de engorda y alimentar con la dieta que sea pertinente para finalizar al animal. Estos pesajes también ayudarán en la selección de las mejores hembras para reproductores futuros.

Los productores poseen un grupo de animales rezagados que son separados del resto debido a que no alcanzan el peso ni la conformación que ellos consideran óptima, por lo que estos animales quedan apartados hasta alcanzar un peso adecuado. Como estos animales difieren en varias generaciones (hasta 3 años) y sexo, se recomienda eliminarlos desde un principio, destinándolos para consumo personal o algún comercio local, ya que ocupan espacio, consumen mucho más alimento y por más tiempo de lo normal, lo que puede llegar a no ser rentable. Además, al estar ambos sexos juntos, se corre el riesgo que las hembras queden preñadas, en el caso que los machos no estén castrados. Considerando que estos animales tienen una velocidad de crecimiento más bajo, no es deseable que se reproduzcan ya que es muy probable que la cría tenga también una baja tasa de crecimiento.

En el momento en que los jabatos recién nacidos comienzan a explorar y separase de sus madres se recomienda pesar a los animales en esta etapa y crear un registro de los nuevos individuos, lo que ayudará a mantener un orden con respecto manejos futuros. Indirectamente, también se podrán evaluar características productivas de las madres y su posterior mantenimiento en el criadero como hembra reproductora. Para ello es importante realizar postura de crotales o algún dispositivo que pueda darle una identificación única a cada animal. Se podrían identificar diferentes camadas con crotales de colores diferentes, para así hacer una fácil identificación visual en terreno. Se recomienda además poner crotales o dispositivos de identificación 
a hembras y machos reproductores, nuevamente para ayudar en el orden de los registros y evitar problemas de consanguinidad.

\section{CONCLUSIONES}

En el Fundo Kutrehue, los manejos productivos realizados en su criadero de jabalíes con destino a producción de carne comercial, siguen los estándares utilizados en Chile según la bibliografía consultada. Las fechas en las que se realizan las etapas de encaste, parición y destete siguen un patrón muy similar a lo recomendado y reportado por los diversos autores, por lo que se concluye que en general las prácticas productivas de manejo para crianza de jabalíes son las comúnmente usadas en un sistema semi-extensivo y bajo las condiciones utilizadas en Chile. Sin embargo, es recomendable mejorar algunas condiciones en el criadero para así poder aumentar la productividad de éste, por lo que las recomendaciones y/o sugerencias más relevantes son la colocación de crotales para tener un reconocimiento individual de cada animal, crear un registro con las fechas de alimentación, pesajes y manejos, y ampliar el mercado de ventas.

\section{REFERENCIAS}

FOURNIER-CHAMBRILLON, CH., MAILLARD, D., FOURNIER, P. 1995. Diet of the wild boar (Sus scrofa L.) inhabiting the Montpellier Garrigue. IBEX Journal of Mountain Ecology 3: 174-179.

INSTITUTO NACIONAL DE ESTADÍSTICA (INE). 2012. http:// www.ine.cl/ (acceso, 20.06.2012).

JABALÍCHILE., 2006. Los orígenes del jabalí. http://www. produccion-animal.com.ar/produccion_jabalies/01origenes_jabali.pdf (acceso, 18.06.2012).

NIXDORF, R., BARBER, L. 2001. Wild Boar Production. Economic and production information for Saskatchewan producers. Saskatchewan Agriculture and Food, Canada. $30 \mathrm{p}$.

NOVOA, P. 2002. Producción de jabalí y sus posibilidades en Chile. Seminario de título. Santiago. Pontificia Universidad Católica de Chile. Departamento de Zootecnia. 14 p.

SKEWES, O., MORALES, R. 2006. Crianza de jabalí (Sus scrofa L.) en Chile. Distribución, tamaño y aspectos básicos de manejo. Agrociencia 22(1): 29-36. 